\title{
Composantes et contrôle de la dépense énergétique cellulaire chez l'endotherme *
}

\author{
AE Chinet \\ Département de physiologie de l'université, CMU, 1, rue Michel-Servet, 1211 Genève 4, Suisse
}

(Reçu le 22 mai 1989; accepté le 17 octobre 1989)

\begin{abstract}
Résumé - Quelques tentatives de prédiction et de mesure du coût énergétique de l'homéostasie ionique cellulaire chez l'endotherme sont passées en revue, et limportance du maintien des flux ioniques transmembranaires - autant que celui des gradients - pour la préservation des structures et fonctions cellulaires est soulignée. Les conséquences énergétiques des ajustements aigus et chroniques de deux processus de transport actif primaire, le transport $\mathrm{Na}-\mathrm{K}$ à travers le plasma(sarco)lemme et le transport de Ca à travers la membrane du réticulum endo(sarco)plasmique sont illustrées. La question de la contribution du cycle hydrolyse-resynthèse des protéines à la dépense énergétique d'entretien cellulaire est posée, mais il semble prématuré d'apporter une réponse quantitative définitive. Après un rappel des concepts aujourd'hui bien établis du contrôle «intrinsèque" de la dépense énergétique cellulaire d'une part, et de son contrôle "extrinsèque" par les activités intracellulaires d'ion calcium d'autre part, il est suggéré que l'organe contribue lui aussi, par la distribution hétérogène du sang artériel et des érythrocytes aux cellules, au contrôle du métabolisme énergétique cellulaire en l'absence d'ischémie.
\end{abstract}

endotherme / métabolisme énergétique d'entretien / homéostasle / sodium / calcium

Summary - Components and control of thermogenesis in the endotherms (endothermogenesis). Attempts to predict and to measure the energy cost of ionic homeostasis in tissue cells are briefly reviewed and attention is drawn to the importance of ion-flux - as well as ion-gradient maintenance for the preservation of cell structure and function. The energetic consequences of acute and chronic adjustments of two primary-active transport processes, Na-K transport across plasma(sarco) lemma and Ca transport across the endo(sarco)plasmic-reticular membrane, are illustrated. The question of the contribution of protein turnover to the energy expenditure of cell maintenance is raised, but not answered. After looking back on the now well-established concepts of the "intrinsic" cellular control of energy expenditure and the so-called "extrinsic" cellular control by intracellular calcium ion activities, it is suggested that the organ may also control cell metabolism under non-ischemic conditions, through heterogeneous distribution of arterial blood and erythrocytes to cells.

endotherms / maintenance metabolic rate / homeostasis / sodium / calcium

\footnotetext{
" Travail présenté à la $5^{\ominus}$ Conférence sur la nutrition et l'alimentation des herbivores, INRA, Paris, 1617 mars 1989.
} 


\section{INTRODUCTION}

L'objet vivant au sens large (flore et faune d'une région, organisme, cellule isolée) est "économe" quant à son entretien dans son biotope. A l'état stationnaire, la quantité d'énergie dissipée (ou la production interne d'entropie) par unité de temps correspondrait en effet à un minimum (Glansdorff et Prigogine, 1971). Ce minimum est nécessaire à l'entretien des structure et fonctions de l'objet vivant qui n'est autre, à l'état stationnaire, que le "catalyseur instable" de l'ensemble des transformations nettes de substrats à produits du métabolisme. Si ce minimum se trouve être plus élevé chez un endotherme que chez un ectotherme, c'est parce que la cellule - ou l'unité structurofonctionnelle - de l'endotherme est un catalyseur globalement plus instable que la cellule de l'ectotherme. Une telle évolution, vers plus d'instabilité structurofonctionnelle, suggère que le monde vivant tend à canaliser toujours plus, à son profit, la production d'entropie planétaire (Morel et Fleck, 1989).

On a longtemps voulu mettre le doigt sur la différence endotherme-ectotherme en cherchant quel mécanisme dissipateur d'énergie pourrait être propre à la cellule d'endotherme, mais on n'a pas trouvé de différence qualitative décisive. Parmi les différences quantitatives, on a relevé la plus grande perméabilité des membranes cellulaires aux ions minéraux (Else et Hulbert, 1987) s'accompagnant, à gradients électrochimiques comparables, de flux stationnaires plus élevés chez l'endotherme. Mais cette différence, liée peut-être au fait que les tissus mous contiennent environ 3 fois moins de calcium et 2 fois moins de magnésium chez l'endotherme que chez l'ectotherme (Diem et Lentner, 1972), n'est probablement pas la seule : c'est apparemment l'ensemble des processus cellu- laires, et non un processus particulier, qui même à température d'observation égale est accéléré chez l'endotherme (5 à 10 fois plus rapide chez l'endotherme que chez l'ectotherme). Tel est le message que la première partie de cette revue voudrait contribuer à transmettre.

La deuxième partie, consacrée au contrôle cellulaire du métabolisme énergétique, est un rappel des concepts établis en même temps qu'une occasion de présenter l'hypothèse d'une contribution de l'organe au contrôle du métabolisme énergétique cellulaire dans le muscle squelettique au repos en conditions physiologiques (c'est-à-dire en l'absence d'ischémie).

\section{COMPOSANTES \\ DE LA THERMOGENĖSE CHEZ L'ENDOTHERME}

\section{Coût énergétique de l'homéostasie lonique}

Les transports actifs primaires de sodium et de potassium par la $\mathrm{Na}, \mathrm{K}$ ATPase de la membrane plasmatique (sarcolemme dans le muscle), et de calcium par la Mg,Ca ATPase de la membrane du réticulum endoplasmique (sarcoplasmique dans le muscle), sont des systèmes capables de fonctionner à haut régime (Clausen, 1986; Hasselbach et Oetliker, 1983), donc susceptibles de consommer beaucoup d'énergie. Même lorsque les flux ioniques passifs à travers les membranes cellulaires sont réduits au minimum (cellule non stimulée), ils doivent être exactement compensés, à l'état stationnaire, par des transports actifs primaires, ou des transports actifs secondaires (c'est-à-dire ayant pour source énergétique les gradients ioniques établis par les transports actifs primaires). II en est ainsi pour tous les mouvements ioniques, 
à travers la membrane mitochondriale interne (MMI) comme à travers les 2 autres membranes cellulaires.

II faut remarquer que si les taux de recirculation ioniques sont réduits dans la cellule non stimulée, ils ne sauraient y être nuls. En effet, les mouvements des ions (et pas seulement leurs gradients) sont indispensables aux transports d'ADP et ATP à travers la MMI par la translocase (Klingenberg, 1980) et le transporteur ATP/P (Aprille, 1988), aux transports des acides pyruvique, citrique/isocitrique, 2-oxoglutarique, succinique, malique, oxalacétique à travers cette même membrane (Reich et Sel'kov, 1981, cités par Förster, 1988), aux transports de glucose (Wright et al, 1986) et d'acides aminés (Moore, 1983; Hundal et al, 1989) à travers la membrane plasmatique.

\section{Transport actif $\mathrm{Na}-\mathrm{K}$}

Il a été suggéré à plusieurs reprises (par exemple, Whittam, 1961) qu'un des transports actifs primaires d'ions minéraux, le transport $\mathrm{Na}-\mathrm{K}$, pourrait être un déterminant majeur de la dépense énergétique cellulaire chez l'endotherme (dépense d'énergie égale, dans l'état stationnaire, à une production de chaleur que l'on appellera ici de façon abrégée «endothermogenèse"). Les auteurs qui ont tenté de calculer, sur la base de mesures de flux ioniques et de différences de potentiel électrochimiques, la part de l'endothermogenèse dont le transport actif $\mathrm{Na}-\mathrm{K}$ est directement responsable ont trouvé entre $2 \%$ (Creese, 1968) et plus de $100 \%$ (Ling, 1984) selon les prémisses qu'ils avaient adoptées.

Les mesures plus directes effectuées sur des muscles de mammifères ont abouti à des estimations comprises entre moins de $10 \%$ et $40 \%$ de la production totale de chaleur du muscle squelettique (Ems) au repos. Les valeurs les plus élevées (Ismail-Beigi et Edelman, 1970; Don Stevens et Kido, 1974; Asano et al, 1976; Horwitz et Eaton, 1977) émanent d'observations des conséquences à long terme d'une inhibition du transport actif $\mathrm{Na}-\mathrm{K}$ sur la respiration cellulaire, ou d'observations faites sur des préparations musculaires dont les fibres n'étaient pas intactes et n'avaient pas encore eu le temps de se réparer au moment où les mesures étaient pratiquées (fig 1). Toutes les observations des effets aigus d'une inhibition du transport actif $\mathrm{Na}-\mathrm{K}$ soit par l'ouabaïne, inhibiteur spécifique de ce transport, soit par la suppression (substitution) du sodium extracellulaire, sur des préparations intactes ou dont les fibres lésées avaient eu le temps de se réparer, ont révélé une diminution de Ems inférieure ou égale à 10\% seulement. L'hypothèse d'un contrôle relativement étroit du métabolisme oxydatif par les ATPases globales étant admise, il en a été déduit que le coût énergétique du transport actif $\mathrm{Na}-\mathrm{K}$ et de toutes ses conséquences métaboliques directes (coût global) était de l'ordre de 10\% de Ems (Chinet et al, 1977; Fagher et al, 1987).

Il a été suggéré que même dans le tissu nerveux, où la surface totale de membrane plasmatique par unité de masse tissulaire est élevée, le coût énergétique global du transport actif $\mathrm{Na}-\mathrm{K}$ avait pu être largement surestimé par Whittam et d'autres, au début des années 60 , parce que les effets à moyen terme d'une inhibition du transport actif $\mathrm{Na}-\mathrm{K}$ par l'ouabaïne sur le métabolisme énergétique sont plus complexes que ces auteurs ne l'avaient envisagé (Swanson, 1968). Schématiquement, si l'interruption du transport actif d'ion sodium entraîne la diminution d'un transport de substrat énergétique dépendant du gradient électrochimique de cet ion, la chute d'intensité du métabolisme peut largement excéder, à moyen terme, celle que l'on 


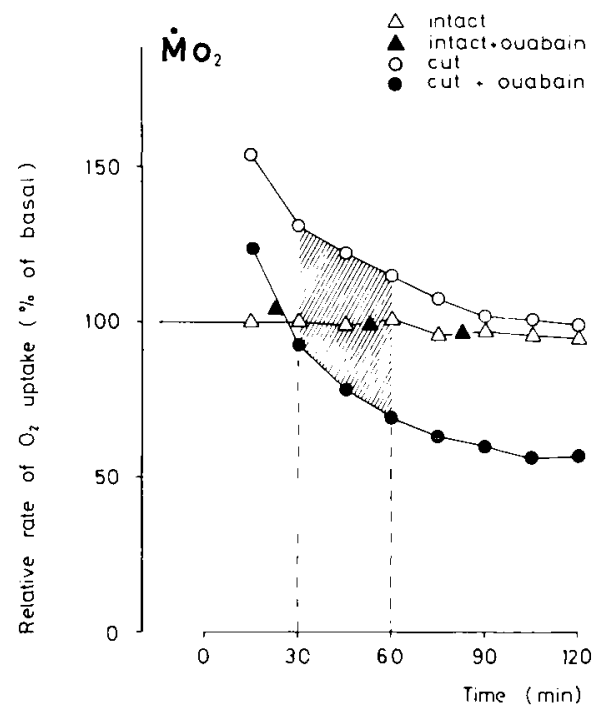

Fig 1. (D'après Biron et al (1979). Effets de l'intégrité cellulaire et de l'inhibition du transport actif Na-K par l'ouabaine $\left(10^{-3} \mathrm{M}\right)$ sur la consommation d'oxygène $\left(\mathrm{MO}_{2}\right)$ relative de 4 groupes de muscles soléaires isolés de souris. Mesures effectuées à $30^{\circ} \mathrm{C}$, en milieu phosphate renouvelé, à partir du moment où les muscles de 2 des groupes (cercles) ont été lésés par coupure transversale des fibres près des extrémités du muscle. Des 2 groupes de muscles exposés à l'ouabaïne dès le temps zéro (symboles pleins), seuls les muscles lésés ont une respiration diminuée par rapport au témoin sans ouabaïne. L'ouabaïne a été appliquée immédiatement après la lésion, c'est-à-dire avant que le processus de réparation, lequel dure environ 2 h, ait pu même commencer.

observe en condition aiguë (c'est-à-dire lorsque seule la dépense énergétique liée au transport de sodium est supprimée). La métaphore suivante, à la fois synthétique et parlante, a été proposée par un lecteur avisé : si l'on constate que le trafic des camions alimentant les citoyens d'une ville varie parallèlement au budget global de ces derniers, on ne va pas en conclure que l'essentiel de leurs dépenses est constitué de frais de transport.

\section{Transport actif de Ca par l'ATPase du réticulum}

On admet généralement que le contrôle de l'activité de calcium cytosolique est assuré, dans la cellule non stimulée, par les processus responsables de la recirculation de $\mathrm{Ca}$ à travers la membrane du réticulum endo(sarco)plasmique, ceci étant vrai même pour le myocarde (Carafoli, 1985). La fraction de Ems dévolue à ce processus homéostatique a été estimée à $7 \%$ dans le muscle squelettique sur la base de données morphométriques et de flux calciques mesurés sur des microvésicules (Hasselbach et Oetliker, 1983).

En utilisant le dantrolénate de sodium comme inhibiteur spécifique de la libération de Ca par le canal calcique du réticulum sarcoplasmique (Martonosi, 1984), nous avons détecté à l'aide des techniques de microcalorimétrie développées dans notre laboratoire une chute de Ems basale (et de la respiration de base du muscle) de l'ordre de 5 à $10 \%$ sur le muscle soléaire de Souris en conditions de superfusion.

\section{Recirculations ioniques à travers la MMI}

La part du transport actif primaire d'ions hydrogène destinée à entretenir les recirculations ioniques (notamment celles de $\mathrm{Ca}$ et $\mathrm{Na}$ ) à travers la $\mathrm{MMI}$ (fig 2) ne saurait expliquer que quelques pourcents de la respiration cellulaire maximum (McCormack et Denton, 1986; Diwan, 1987). II faut toutefois remarquer que ce maximum correspond à un flux d'énergie égal à un cinquième environ de Ems au repos.

Un moyen de limiter le taux de recirculation ionique à travers la MMI dans la cellule intacte est d'élever l'activité de $\mathbf{M g}$ 


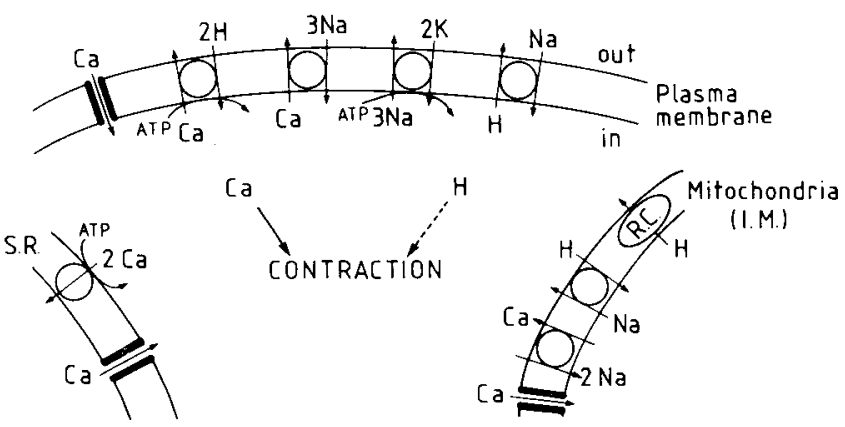

Fig 2. (D'après McCormack et al (1988). Principaux mouvements ioniques dans la cellule cardiaque. Les charges sur les ions ont été omises pour plus de clarté. RC : chaîne respiratoire; IM : membrane mitochondriale interne; SR : réticulum sarcoplasmique. La recirculation des lons $\mathrm{Ca}, \mathrm{Na}$ et $\mathrm{H}$ à l'état stationnaire à travers la membrane mitochondriale interne est susceptible de dissiper une certaine quantité d'énergie par unité de temps.

cytosolique (McCormack et Denton, 1986), laquelle est normalement inférieure à $1 \mathrm{mM}$ (Garfinkel et Garfinkel, 1984; Murphy et al, 1989). Ceci peut être réalisé simplement en augmentant la concentration extracellulaire de $\mathrm{Mg}$. Lorsque celle-ci est augmentée de $10 \mathrm{mM}$ (c'est-à-dire à 11,2 $\mathrm{mM}$ ), Ems et respiration basale diminuent davantage que sous l'effet du dantrolénate. Ceci laisse supposer qu'une partie de l'endothermogenèse, dans le muscle au repos, pourrait provenir de la recirculation d'ions à travers la MMI. Toutefois, cette contribution est probablement modeste parce qu'une élévation artificielle de $\mathrm{Mg}$ intracellulaire inhibe la libération de $\mathrm{Ca}$ du réticulum (Hymel et al, 1988) aussi bien que l'entrée électrophorétique de $\mathrm{Ca}$ dans la matrice mitochondriale. C'est donc à la seule différence entre les effets aigus du $\mathrm{Mg}$ et du dantrolénate sur l'endothermogenèse que se limiterait cette contribution (quelques pourcents de Ems dans la cellule non stimulée).

\section{Coût énergétique du cycle de resynthèse des protéines}

Une fraction importante de l'endothermogenèse est sans doute associée au cycle de renouvellement des protéines cellulaires. Selon quelques sources récentes, cette fraction s'élèverait à $20-27 \%$ chez le mammifère (Webster, 1983; Nair et al, 1988), comme chez le poulet à l'état nourri (Aoyagi et al, 1988). A noter que dans le dernier travail cité, l'estimation était fondée sur des mesures de taux de synthèse des protéines et de production de chaleur de l'animal entier, avant et après administration de cycloheximide (un inhibiteur de la synthèse des protéines).

\section{Conclusion provisoire}

Si le coût énergétique de l'homéostasie ionique dans la cellule d'endotherme au 
repos ne dépasse pas $25 \%$ du métabolisme énergétique (10\% pour le transport $\mathrm{Na}-\mathrm{K}, 10 \%$ pour l'homéostasie du calcium cytosolique et au maximum $5 \%$ pour l'homéostasie du calcium dans la matrice mitochondriale), et si le cycle de renouvellement des protéines ne dissipe pas plus d'énergie que l'homéostasie ionique, alors il reste à trouver l'origine - ou les processus dissipateurs d'énergie responsables - de $50 \%$ de l'endothermogenèse dans le muscle squelettique au repos.

\section{Ajustements aigus des taux de recirculation ioniques à travers les 3 membranes cellulaires}

Afin de montrer que le coût énergétique modeste de l'homéostasie ionique d'entretien est compatible avec l'apparition de dépenses énergétiques bien plus importantes lorsque les transports ioniques sont sollicités, quelques cas particuliers sont présentés: a) activation de transports actifs secondaires, dont l'échangeur $\mathrm{Na} / \mathrm{H}$ de la membrane plasmatique, sous l'effet d'une contrainte hyperosmotique, avec activation concomitante du transport actif primaire $\mathrm{Na}-\mathrm{K}$ (Chinet et Giovannini, 1989); b) activation plus directe du transport $\mathrm{Na}-\mathrm{K}$ par un ionophore du sodium, l'antibiotique monensine (Chinet, 1989); c) activation de la recirculation de $\mathrm{Ca}$ à travers la membrane du réticulum sarcoplasmique - et probablement aussi la MMI - sous l'effet d'augmentations modestes de la concentration du potassium extracellulaire (Chinet et Giovannini, 1988). Les coûts énergétiques des 2 composantes de l'homéostasie ionique, la mitochondriale et l'extramitochondriale (cette dernière étant aussi en partie, mais indirectement, mitochondriale), sont difficiles à évaluer expérimentalement parce que le moyen que l'on a, sur la cellule entière, d'inhiber l'une (haut $\mathrm{Mg}$ ) inhibe aussi l'autre (Hymel et al, 1988), et qu'une stimulation de la composante mitochondriale par une élévation du $\mathrm{pH}$ cytoplasmique (par exemple, Heaton et Nicholls, 1976) stimule aussi la composante extramitochondriale (Grassi de Gende, 1988).

\section{Ajustements lents des taux de recirculation ioniques accompagnant des modifications de l'endothermogenese}

Les hormones thyroïdiennes, comme aussi l'acclimatation au froid, induisent apparemment un accroissement des taux de recirculation ioniques à travers la MMI (Hafner et al, 1988; Himms-Hagen et al, 1976), et également à travers les autres membranes cellulaires. Une augmentation de perméabilité de la membrane plasmatique et/ou du réticulum qui, secondairement, entraînerait une augmentation de la capacité du transport actif $\mathrm{Na}-\mathrm{K}$ (Everts et Clausen, 1988) et du transport actif de Ca par le réticulum sarcoplasmique (Simonides et Van Hardeveld, 1989) est observée sous l'effet des hormones thyroïdiennes. Les conséquences énergétiques de ces ajustements lents de la recirculation ionique ont souvent été décrites en mettant l'accent sur un accroissement de la part ouabaïnesensible de la respiration cellulaire. Cette part est augmentée de $90 \%$ dans le muscle par l'acclimatation au froid, mais il est bon de réaliser que, relativement au flux métabolique cellulaire total, elle augmente de moins de 50\% (Don Stevens et Kido, 1974; Horwitz et Eaton, 1977; Herpin et al, 1987). La différence entre valeurs absolue et relative est encore plus marquée lors de comparaisons d'état thyroïdiens : la valeur absolue entre hypo- et hyperthyroidiens augmente de $190 \%$ mais la valeur relative de $35 \%$ seulement (c'est-à-dire de 
$6 \%$ à $8 \%$ de Ems selon Biron et al, 1979). Les accroissements de $155 \%$ et $150 \%$ de la part dantrolénate-sensible de Ems entre l'hypo- et l'euthyroïdien et entre l'hypo- et I'hyperthyroïdien (Van Hardeveld et Clausen, 1984) deviennent eux aussi, relativement à Ems total, beaucoup plus modestes ( $7 \%$ et $-9 \%$ respectivement). Enfin, nous n'avons pas observé d'accroissement significatif des fractions de Ems suppressibles par le dantrolénate ou le haut $\mathrm{Mg}$ dans le muscle soléaire de souris entre les températures d'acclimatation de $\mathbf{3 4}$ et de $5^{\circ} \mathrm{C}$ (en préparation).

\section{Conclusion de la 1re partie}

Whittam (1961) suggérait que le transport ionique à travers les membranes cellulaires agit en tant que "pacemaker" de l'activité métabolique. Eu égard au fait que les recirculations ioniques, bien que peu coûteuses du point de vue énergétique, jouent un rôle primordial dans le transport des substrats énergétiques dont dépend tout le métabolisme cellulaire, il semble judicieux de conserver la métaphore du "pacemaker». L'intensité du métabolisme énergétique paraît bien, fonctionnellement sinon énergétiquement, étroitement corrélée à celle des recirculations ioniques.

\section{CONTRÓLE \\ DE L'ENDOTHERMOGENĖSE CELLULAIRE}

\section{Contrôle intrinsèque}

Depuis l'établissement du modèle selon lequel une diminution de concentration d'ADP est le principal frein de la respiration cellulaire (Chance et Williams, 1956), d'autres modèles sont apparus dans les- quels [ADP] conserve un rôle clé (contrôles de la respiration par le potentiel phosphate; le rapport [ATP]/[ADP]; le potentiel phosphate et une diminution du rapport NADH/NAD mitochondrial). Dans tous ces modèles et d'autres encore plus compliqués (par exemple celui qui définit l'intensité de la respiration cellulaire en fonction de la puissance trois du rapport [ADP] [Pi]/[ATP], du logarithme du rapport NADH/ NAD et de la racine carrée de la concentration d'oxygène) on trouve toujours, sous-jacente, l'idée que l'activité des AT-Pases globales détermine, en tant que fournisseur d'ADP, l'intensité du métabolisme énergétique (concept du contrôle intrinsèque de la respiration, cf. Denton et McCormack, 1980).

\section{Contrôle extrinsèque}

L'observation d'une dissociation entre l'activité respiratoire et la rephosphorylation d'ADP dans des états physiologiques (l'exemple le plus spectaculaire est la stimulation adrénergique de la cellule de tissu adipeux brun), ainsi que d'autres observations tendant à confirmer la théorie chémiosmotique de la phosphorylation oxydative, ont fait apparaître le potentiel d'oxydoréduction des substrats de la respiration comme un déterminant de la respiration cellulaire relativement indépendant du potentiel phosphate. Ainsi les contrôles de la respiration cellulaire par limitation de la disponibilité en substrats (Akerboom et al, 1978), de la perméabilité de la MMI à certains substrats, et enfin de l'activité de certaines deshydrogénases médiée par les processus responsables de l'homéostasie du calcium dans la matrice mitochondriale (Denton et McCormack, 1985; Hansford, 1985) pourraient-ils être appelés, globalement, "contrôle extrinsèque» de la respiration (Denton et McCormack, 1980; McCor- 
mack et Denton, 1988). Enfin, un contrôle de la respiration cellulaire par limitation de la disponibilité en oxygène, si un tel contrôle existe physiologiquement (voir dernier sous-titre), devrait lui aussi être considéré comme un des facteurs multiples de contrôle extrinsèque.

\section{Contrôle de l'accès aux cellules des flux de substrats à l'organe}

Si l'on appelle cflux de substrat à un organe" le produit du débit sanguin à l'organe par la concentration sanguine de l'espèce chimique (c'est-à-dire de delivery des Anglo-Saxons), alors on peut clairement distinguer les états physiologiques normaux, où les flux de substrats à l'organe ne sont pas des facteurs limitants du métabolisme énergétique, d'un état d'ischémie. En effet, dans l'ischémie, les flux de substrats, notamment celui d'oxygène, sont anormalement réduits et il y a également réduction des débits d'élimination de produits du métabolisme, notamment d'acide non volatil provenant du catabolisme anaérobie. On a aujourd'hui de bonnes raisons de croire que dans le muscle normal au travail, même au voisinage de la consommation maximum d'oxygène, non seulement le flux d'oxygène à lorgane mais aussi la répartition de ce flux aux cellules ne sont pas des facteurs limitants de la respiration (Gayeski et al, 1987; Schwertzmann et al, 1989). Il en est tout autrement pour le muscle normal au repos (Chinet et Mejsnar, 1989), où les flux de substrats à l'organe ne sont pas limitants mais où, en revanche, à cause d'une distribution inégale de ces flux au sein de l'organe, leur accès aux cellules est limité. Pour le flux d'oxygène, la cause en est vraisemblablement une hétérogénéité de distribution des érythrocytes dans la microcirculation (Meyer et al, 1987; Ley et al, 1988; Duling et Damon, 1987). II a été démontré qu'une telle hétérogénéité disparaît dès que le muscle est stimulé (Honig et al, 1982). Elle pourrait aussi être supprimée, artificiellement, par perfusion du muscle à haut débit avec une solution oxygénée ne contenant pas d'érythrocytes, procédé qui entraîne une augmentation de la respiration du muscle au repos à des valeurs 3 fois supérieures à celle du muscle autoperfusé au sang (Chinet et Mejsnar, 1989). L'hypothèse explicative d'un tel résultat, laquelle reste à tester, est que l'augmentation artificielle de l'accès aux cellules des flux de substrats à l'organe entraîne une augmentation des potentiels énergétiques cellulaires propre à accroître la dissipation d'énergie nécessaire à l'entretien du nouvel état structuro-fonctionnel cellulaire. II faudrait alors considérer que dans les muscles au repos en conditions physiologiques, chez un animal n'ayant aucune thermogenèse régulatoire à fournir, l'organisme réalise une économie d'énergie en maintenant, par un contrôle extrinsèque de la respiration cellulaire à l'échelon de l'organe, des potentiels énergétiques cellulaires inférieurs à ceux que l'on peut observer en l'absence des limitations d'apport d'oxygène ou de substrats, ou d'élimination des produits du métabolisme.

Parmi les nombreux faits compatibles avec une telle hypothèse, on relèvera qu'une disponibilité accrue en substrats et en oxygène peut non seulement accroître le taux des recirculations ioniques à travers la MMl et l'activité de certaines enzymes-clé du cycle de Krebs (Stucki, 1976; Brown et Brand, 1986; Holness et Sugden, 1989), mais encore augmenter le taux de renouvellement des protéines cellulaires. En effet, une sensibilité jusqu'ici non reconnue du protéasome à la concentration d'ATP dans le domaine physiologique a été récemment mise en évidence (Driscoll et Goldberg, 1989). Une telle sen- 
sibilité pourrait contribuer à expliquer l'effet de l'état nutritionnel de l'animal sur le coût énergétique de l'entretien de ses protéines (Aoyagi et al, 1988).

\section{Conclusion de la $2 e$ partie}

Le contrôle de la dissipation d'énergie cellulaire chez l'endotherme (et aussi chez l'ectotherme) est certainement un phénomène multifactoriel. Au contrôle exercé en commun par le rapport [ATP]/[ADP] — ou le potentiel phosphate - et le rapport NAD/NADH mitochondrial (contrôle intrinsèque), s'ajoute le contrôle par l'activité de $\mathrm{Ca}$ dans la matrice mitochondriale (contrôle extrinsèque). Ce dernier étant luimême étroitement lié aux contrôles exercés par la disponibilité en substrats (notamment en oxygène), une limitation par l'organe de l'accès des substrats aux cellules (hétérogénéité de distribution, au sein de l'organe, de flux non limitants de substrats à l'organe) pourrait être partie intégrante du contrôle extrinsèque de la respiration cellulaire.

Dans l'ensemble, les études de physiologie cellulaire comparée ont fourni des données quantitatives qui témoignent d'une différence entre l'économie énergétique des endothermes et celle des ectothermes, sans toutefois nous faire entrevoir un mécanisme ponctuel à l'origine de cette différence.

\section{RÉFÉRENCES}

Akerboom PM, Bookelman H, Zuurendonk PF, Van der Meer R, Tager JM (1978) Intramitochondrial and extramitochondrial concentrations of adenine nucleotides and inorganic phosphate in isolated hepatocytes from fasted rats. Eur J Biochem 84, 413-420
Aoyagi Y, Tasaki I, Okumura JI, Muramatsu T (1988) Energy cost of whole-body protein synthesis measured in vivo in chicks. Comput Biochem Physiol 91 A, 765-768

Aprille JR (1988) Regulation of the mitochondrial adenine nucleotide pool size in liver: mechanism and metabolic role. Fed Am Soc Exp Biol J 2, 2547-2556

Asano Y, Liberman UA, Edelman IS (1976) Relationships between $\mathrm{Na}$-dependent respiration and $\mathrm{Na}+\mathrm{K}$-adenosine triphosphatase activity in rat skeletal muscle. J Clin Invest 57, 368-379

Biron R, Burger A, Chinet A, Clausen T, DuboisFerrière R (1979) Thyroid hormones and the energetics of active sodium-potassium transport in mammalian skeletal muscles. $J$ Physiol 297, 47-60

Brown GC, Brand MD (1986) Changes in permeability to protons and other cations at high proton motive force in rat liver mitochondria. Biochem J 234, 75-81

Carafoli $E$ (1985) The homeostasis of $\mathrm{Ca}$ in heart cells. J Mol Cell Cardiol 17, 203-212

Chance B, Williams GR (1956) The respiratory chain and oxidative phosphorylation. Adv Enzymol 17, 65-134

Chinet A (1989) Energy cost of ionic homeostasis in mammalian skeletal muscle. In: Energy Transformations in Cells and Organisms. (Wieser W, Gnaiger E, eds) Georg Thieme, Stuttgart, 58-65

Chinet A, Giovannini P (1988) Calciumdependent energy expenditure in the relaxed soleus muscle of the mouse. Experientia 44, A33

Chinet A, Giovannini P (1989) Evidence by calorimetry for an activation of sodium/hydrogen exchange of young rat skeletal muscle in hypertonic media. J Physio/ 415, 409-422

Chinet A, Mejsnar J (1989) Is resting muscle oxygen uptake controlled by oxygen availability to cells? J App/ Physiol 66, 253-260

Chinet A, Clausen T, Girardier L (1977) Microcalorimetric determination of energy expenditure due to active sodium-potassium transport in the soleus muscle and brown adipose tissue of the rat. J Physiol 265, 43-61 
Clausen $T$ (1986) Regulation of active $\mathrm{Na}-\mathrm{K}$ transport in skeletal muscle. Physiol Rev 66, $542-574$

Creese $R$ (1968) Sodium fluxes in diaphragm muscle and the effects of insulin and serum proteins. J Physiol 197, 255-278

Denton RM, McCormack JG (1980) On the role of the calcium transport cycle in heart and other mammalian mitochondria. Fed Eur Biol Soc Lett 119, 1-8

Denton RM, McCormack JG (1985) Ca2+ transport by mammalian mitochondria and its role in hormone action. Am J Physiol 249, E543E554

Diem K, Lentner C (1972) Tables scientifiques. Ciba-Geigy SA, Bâle, Suisse, 520-524

Diwan JJ (1987) Mitochondrial transport of $\mathrm{K}^{+}$ and $\mathrm{Mg}^{+}$. Biochim Biophys Acta 895, 155165

Don Stevens E, Kido M (1974) Active sodium transport: a source of metabolic heat during cold adaptation in mammals. Comput Biochem Physiol 47A, 395-397

Driscoll J, Goldberg AL (1989) Skeletal muscle proteasome can degrade proteins in an ATPdependent process that does not require ubiquitin. Proc Natl Acad Sci USA 86, 787-791

Duling BR, Damon DH (1987) An examination of the measurement of flow heterogeneity in striated muscle. Circul Res 60, 1-13

Else PL, Hulbert AJ (1987) Evolution of mammalian endothermic metabolism: "leaky" membranes as a source of heat. Am J Physiol 253, R1-R7

Everts ME, Clausen T (1988) Effects of thyroid hormone on Na-K transport in resting and stimulated rat skeletal muscle. Am J Physiol 255, E604-E612

Fagher B, Sjögren A, Monti M (1987) A microcalorimetric study of the sodium-potassium pump and thermogenesis in human skeletal muscle. Acta Physiol Scand 131, 355-360

Förster MEC (1988) Citric acid cycle as a "onestep" reaction. J Theoret Biol 133, 1-11

Garfinkel L, Garfinkel D (1984) Calculation of free- $\mathrm{Mg}^{2+}$ concentration in adenosine $5^{\prime}$ triphosphate containing solutions in vitro and in vivo. Biochemistry 23, 3 547-3 552

Gayeski TEJ, Connett RJ, Honig CR (1987) Minimum intracellular $\mathrm{PO}_{2}$ for maximum cy. tochrome turnover in red muscle in situ. Am J Physiol 252, H906-H915

Glansdorff P, Prigogine I (1971) Structure, stabilité et fluctuations. Masson, Paris

Grassi De Gende AO (1988) The effect of $\mathrm{pH}$ on the calcium dependence of calcium accumulation in dog cardiac muscle sarcoplasmic reticulum. J Mol Cell Cardiol 20, 1 087-1 093

Hafner RP, Nobes CD, McGown AD, Brand MD (1988) Altered relationship between protonmotive force and respiration rate in nonphosphorylating liver mitochondria isolated from rats of different thyroid hormone status. Eur J Biochem 178, 511-518

Hansford RG (1985) Relation between mitochondrial calcium transport and control of energy metabolism. Rev Physiol Biochem Pharmacol 102, 2-66

Hasselbach W, Oetliker H (1983) Energetics and electrogenicity of the sarcoplasmic reticulum calcium pump. Annu Rev Physiol 45, 325-339

Heaton GM, Nicholls DG (1976) The calcium conductance of the inner membrane of rat liver mitochondria and the determination of the calcium electrochemical gradient. Biochem J 156, 635-646

Herpin PR, McBride BW, Bayley HS (1987) Effect of cold exposure on energy metabolism in the young pig. Can J Physiol Pharm 65, 236-345

Himms-Hagen J, Behrens W, Hbous A, Greenway D (1976) Altered mitochondria in skeletal muscle of cold acclimated rats and the adaptation for nonshivering thermogenesis. In: Regulation of Depressed Metabolism and Thermogenesis. (Jansky L, Musacchia XJ, eds), Charles G Wilber, Springfield, Illinois, USA, 243-260

Holness MJ, Sugden MC (1989) Pyruvate dehydrogenase activities during the fed-tostarved transition and on re-feeding after acute or prolonged starvation. Biochem $J$ 258, 529-533

Honig CR, Odoroff CL, Frierson JL (1982) ACtive and passive capillary control in red muscle at rest and in exercise. Am J Physiol 243, $\mathrm{H}+96-\mathrm{H} 206$

Horwitz BA, Eaton M (1977) Ouabain-sensitive liver and diaphragm respiration in cold- 
acclimated hamster. J Appl Physiol 42, 150153

Hundal HS, Rennie MJ, Watt PW (1989) Characteristics of acidic, basic and neutral amino acid transport in the perfused rat hindlimb. $J$ Physiol 408, 93-114

Hymel L, Makoto I, Fleischer S, Schindler H (1988) Purified ryanodine receptor of skeletal muscle sarcoplasmic reticulum forms Caactivated oligomeric $\mathrm{Ca}$ channels in planar bilayers. Proc Natl Acad Sci USA 85, 441-445

Ismail-Beigi F, Edelman LS (1970) Mechanism of thyroid calorigenesis: role of active sodium transport. Proc Natl Acad Sci USA 1071 1078

Klingenberg $M(1980)$ The ADP-ATP translocation in mitochondria, a membrane potential controlled transport. J Membr Biol 56, 97-105

Ley K, Lindbom L, Arfors KE (1988) Haematocrit distribution in rabbit tenuissimus muscle. Acta Physiol Scand 132, 373-383

Ling GN (1984) In: Search of the Physical Basis of Life. Plenum Press, New York and London, 411-414

Martonosi AN (1984) Mechanisms of $\mathrm{Ca}^{2}+$ release from sarcoplasmic reticulum of skeletal muscle. Physiol Rev 64, 1 240-1 320

McCormack JG, Denton RM (1986) $\mathrm{Ca}^{2+}$ as a second messenger within mitchondria. Trends Biochem Sci 258-262

McCormack JG, Denton RM (1988) The regulation of mitochondrial function in mammalian cells by $\mathrm{Ca}^{2+}$ ions. Biochem Soc Trans 16 , 523-527

McCormack JG, Boyett MR, Jewell BR, Orchard $\mathrm{CH}$ (1988) lon movement and contractility in heart cells. Trends Pharmacol Sci 9, 343-345

Meyer JU, Lindbom L, Intaglietta M (1987) Coordinated diameter oscillations at arteriolar bifurcations in skeletal muscle. Am $J$ Physiol 253, H568-H573

Moore RD (1983) Effects of insulin upon ion transport. Biochim Biophys Acta 737, 1-49

Morel RE, Fleck G (1989) Onsager's principle: a unifying bio-theme. $J$ Theoret Biol 136, 171175
Murphy E, Steenbergen C, Levy LA, Raju B, London RE (1989) Cytosolic free magnesium levels in ischemic rat heart. J Biol Chem 264, $5622-5627$

Nair KS, Halliday D, Griggs RC (1988) Leucine incorporation into mixed skeletal muscle protein in humans. Am J Physiol 254, E208E213

Reich JG, Sel'Kov EE (1981) Energy Metabolism of the Cell. A Theoretical Treatise. Academic Press, 1-339

Schwertzmann K, Hoppeler H, Kayar SR, Weibel ER (1989) Oxidative capacity of muscle and mitochondria: correlation of physiological, biochemical, and morphometric characteristics. Proc Natl Acad Sci USA 86, 15831587

Simonides WS, Van Hardeveld C (1989) The postnatal development of sarcoplasmic reticulum $\mathrm{Ca}$ transport activity in skeletal muscle of the rat is critically dependent on thyroid hormone. Endocrinology 124, 1 145-1 153

Stucki JW (1976) Efficiency of oxidative phosphorylation and energy dissipation by $\mathrm{H}+$ ion recycling in rat-liver mitochondria metabolizing pyruvate. Eur J Biochem 68, 551-562

Swanson PD (1968) Effects of ouabain on acidsoluble phosphates and electrolytes of isolated cerebral tissues in presence or absence of calcium. J Neurochem 15, 57-67

Van Hardeveld C, Clausen T (1984) Effect of thyroid status on K-stimulated metabolism and $45 \mathrm{Ca}$ exchange in rat skeletal muscle. Am J Physiol 247, E421-E430

Webster AJF (1983) Energetics of maintenance and growth. In: Mammalian Thermogenesis. (Girardier L, Stock M, eds) Chapman \& Hall, London, New York

Whittam R (1961) Active cation transport as a pace-maker of respiration. Nature 4788 , 603-604

Wright JK, Seckler R, Overath P (1986) Molecular aspects of sugar: ion cotransport. Ann Rev Biochem 55, 225-248 